\title{
Assessing Mortality of Released or Discarded Fish Using a Logistic Model of Relative Survival Derived from Tagging Data
}

\author{
Robert E. Hueter, * Charles A. Manire, and John P. Tyminski \\ Center for Shark Research, Mote Marine Laboratory, 1600 Ken Thompson Parkway, \\ Sarasota, Florida 34236, USA \\ John M. Hoenig and Daniel A. Hepworth \\ Virginia Institute of Marine Science, College of William and Mary, Post Office Box 1346, \\ Gloucester Point, Virginia 23062, USA
}

\begin{abstract}
The mortality of released or discarded fish in commercial and recreational fisheries and in fisheries-independent research is a critical area of study for contemporary fisheries science. Key questions involving postrelease survival include the following: What fraction of the bycatch discarded in a commercial fishery survives? What is the hooking mortality of fish released by recreational anglers, and how does this vary with the treatment and condition of the fish? What fraction of tagged fish do not survive the stress of capture and tagging? What percentage of hatchery-raised fish survive after stocking? To address these and related questions, we develop a two-step approach to estimating absolute postrelease survival rates. First, fish are tagged and assigned to discrete classes based on their condition (from best to worst) at release. The relative survival of fish released in different conditions is estimated from tag returns by fitting a logistic model. Then, assuming that fish in the best condition survive to the same degree as fish that were not captured, absolute survival rates in the other categories are determined. Applying this method to one example from field research, we estimate that $69 \%$ of blacktip sharks Carcharhinus limbatus and $60 \%$ of bonnetheads Sphyrna tiburo survived the stress of gill-net capture, tagging, and release in a 1992-2004 study in Florida. Our method has broad application to determining the condition- or treatment-specific survival rates of released fish of many types in fisheries and research projects in which tag-and-recapture methods can be used.
\end{abstract}

The fate of released fish is of general interest in many aspects of fisheries research. In commercial fisheries, a large portion of the catch may be discarded at sea (Alverson et al. 1994), and our ability to assess the status of a stock depends in part on our knowledge of the fate of discards. This is particularly important in determining a fishing operation's total bycatch mortality, which consists of the immediate, at-the-boat, observable mortality plus the postrelease, cryptic mortality caused by the catch-and-release event. Sport fishers are often encouraged or compelled to release a portion of their catch for conservation reasons, and it is important to know to what extent these released fish survive. It also is of interest to know what factors affect their survival. Thus one might ask, for example, whether fish that are hooked in the stomach have a reasonable chance of surviving or whether puncturing the swim bladder improves the chances of survival (Keniry et al. 1996). In some cases, it may be of interest to determine the relative survival of two groups

\footnotetext{
* Corresponding author: rhueter@mote.org
}

Received February 23, 2005; accepted November 30, 2005 Published online April 18, 2006 of fish that have received different treatments, that have been exposed to different conditions, or that have different traits. Relative survival may also be studied because it is easier to estimate relative rates than absolute rates.

There are at least two other situations in which determining the absolute or relative survival rates of released fish is important. In tagging studies, it is generally of interest to determine the fraction of the released fish that survive the initial stress of capture, handling, tagging, and release, so that the actual number of tagged fish in the postrelease experiment is known. In fish stocking operations, it is of interest to determine the survival rate of released fish and how survival is affected by various factors such as genetics or hatchery treatment.

The fate of released fish, however, is poorly known in most cases (Alverson et al. 1994). It can be studied by holding fish in pens for a few days, but there is the potential for artificial conditions in the holding facilities to influence the outcome. For example, animals held in pens may be protected from predators, or they may not consume the same amount of food that they would if they were released into the wild. The 
survival of released fish may also be studied using telemetry techniques (e.g., Heupel and Simpfendorfer 2002). It may be difficult to achieve adequate sample sizes and to track animals for long periods of time, however, and the telemetry equipment may produce an artifact by affecting the survival of the outfitted fish.

In this paper we describe a new approach to quantifying the fate of released fish based on comparing rates of tag returns from fish released in different condition categories. This study was originally motivated by a need to quantify the bycatch mortality of small sharks caught in gill nets in Florida waters prior to the state's banning of this commercial gear type in 1994. The approach has broad application to other gear types and most species of fish for which the condition upon release can be classified into discrete, observable, repeatable categories.

We develop a model describing how the relative abundance of two groups (e.g., fish in two different conditions upon release) evolves over time when they experience a constant ratio of survival rates and show that this model is a logistic regression model. We then consider an important special case in which the two groups have different survival rates at the beginning of the study but equal survival after a period of time, such as after recovery from a capture-release event. In this case, estimation of the relative survival during the initial period of time consists of the simple calculation of the ratio of two ratios. Finally, we examine the case of the relative survival rates of coastal sharks that were captured, tagged, and released in various condition categories. By means of this example, we show how absolute survival estimates can be obtained for many species of fish using our method if it can be assumed that the fish released in the best condition category survive at the same rate as fish that were not captured. In some applications, there may not be a best-condition category that meets the assumption of normal survival (for example, fish with physoclistous swim bladders may all suffer barotraumas when brought to the surface from great depths). In these cases, one can still compute relative survival rates and get a maximal estimate of the overall survival of released fish.

\section{Modeling Relative Survival}

\section{Methods}

We begin by developing deterministic models to describe the relationships between mortality rates, catchability, and tag returns. We then present statistical models for parameter estimation. Suppose that a cohort of animals is caught, tagged, and released, and that each animal is assigned to one of two possible condition classes at the time of release: condition 1 , in which the animal appears perfectly normal; and condition 2 , in which the animal exhibits some specific sign of stress.

Consider first the simple case in which the two categories of animals experience differential survival rates immediately after release, with animals in condition 1 having the higher rate. After a short period of time, typically referred to as the recovery period, the survival rates of the two groups become equal. That is, the stress of capture is a short-term phenomenon, and if an animal survives the first few hours or days after being caught, tagged, and released, it will experience the normal survival rate thereafter. In this case, the ratio of the abundance of condition- 2 to condition- 1 animals
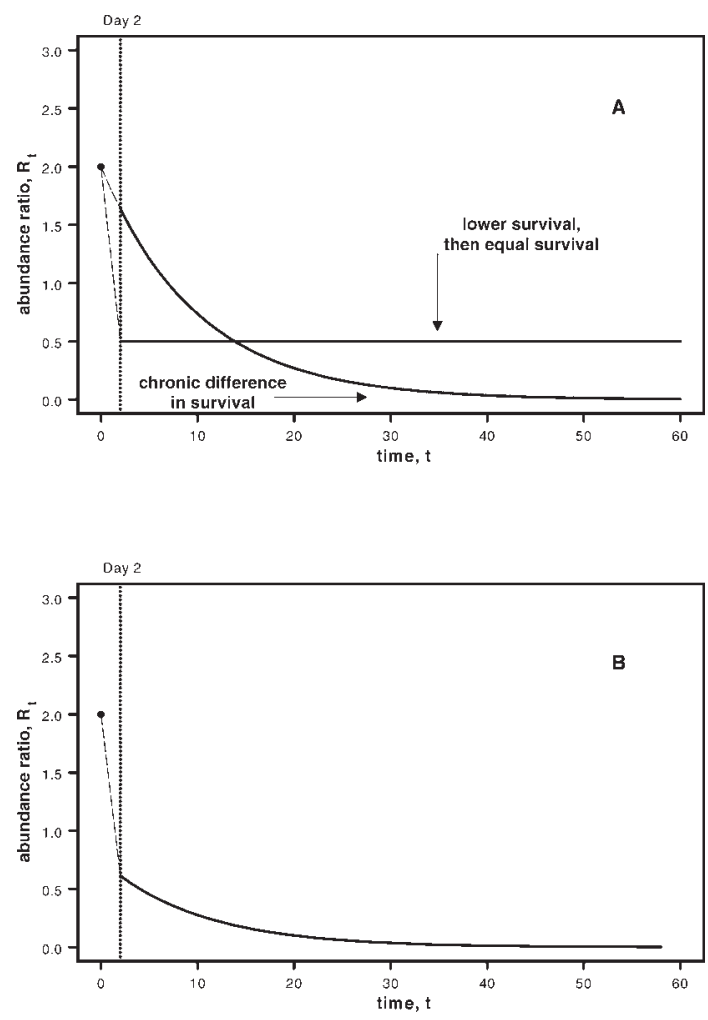

FIGURE 1.-Evolution over time of the ratio of abundances of condition-2 and condition-1 animals when (A) differential mortality due to stress operates only over a 2-d period (kinked line) or continuously at a constant level (smooth curve) or (B) a high differential mortality operates for $2 \mathrm{~d}$, followed by a lower but constant, continuous differential mortality. Dashed lines indicate what happens in the first $2 \mathrm{~d}$ after release. The initial ratio of abundances (2.0) is arbitrary and for illustrative purposes only, as are the drop to 0.5 over a 2 -d period and the degree of curvature of the lines. 
will drop soon after release and then remain stable over time (Figure 1A).

An alternative situation is one in which stressed animals experience a long-term reduction in survival rate. An example might be fish with damaged gills that experience chronic pathologies as a result of their injuries. If the ratio of the survival rates of the animals in the two condition classes $\left(S_{2} / S_{1}\right)$ remains constant over time and the ratio of the numbers of animals released in condition 2 versus condition 1 is $R$, then the ratio of abundance at time $t>0$ is given by

$$
R_{t}=R\left(\frac{S_{2}}{S_{1}}\right)^{t}
$$

(Figure 1A). Here, the survival rates are expressed as finite rather than instantaneous rates.

A more general model allowing for a short-term initial difference in survival followed by a chronic difference is given by

$$
R_{t}=\beta R\left(\frac{S_{2}}{S_{1}}\right)^{t-\delta}, \quad(t>\delta)
$$

where $\beta$ is the initial relative survival rate (Figure $1 \mathrm{~B}$ ) and $\delta$ is the length of the recovery period. A little care is required in defining the time-related variables in this equation. The parameter $\beta$ determines the change in the ratio of abundances over the time period $\delta$ immediately following the release of tagged fish, and the variable $t$ measures time elapsed from time $=0$. Therefore, the chronic difference in mortality operates over a time duration of $t-\delta$.

\section{A Model with Long-Term Effects}

Consider next a discrete-time model where there is a chronic and constant differential in survival between the two groups. Assume that the number of tagged fish caught in a short time interval $(\Delta t)$ is proportional to the amount of sampling (fishing) effort and the abundance of tagged fish. Let the catch of animals in the $t$ th time interval that were released in condition class $i(i=1,2)$ be $C_{i t}$. Then

$$
C_{i t}=q_{i t} f_{t} \Delta t N_{i} \prod_{h=0}^{t-1} S_{i h},
$$

where $q_{i t}$ is the catchability coefficient for animals in condition class $i$ in the $t$ th time interval, $f_{t}$ is the sampling (fishing) effort in that interval, $N_{i}$ is the number of tagged animals released in condition class $i$, and $S_{i h}$ is the finite survival rate of animals released in condition class $i$ in the $h$ th time interval, with $S_{i 0}$ defined to be 1.0. (The catch also depends on the tagreporting rate, but this can be subsumed into the catchability coefficients.) Equation (3) holds when $\Delta t$ is chosen to make the product $q_{i t} f_{t}$ small (Ricker 1975). Hence, at time $t$ the proportion of recaptured animals from condition class 1 is given by

$$
\begin{gathered}
\operatorname{Prop}(1, t)=\frac{C_{1 t}}{C_{1 t}+C_{2 t}} \\
=\frac{q_{1 t} N_{1} \prod_{h=0}^{t-1} S_{1 h}}{q_{1 t} N_{1} \prod_{h=0}^{t-1} S_{1 h}+q_{2 t} N_{2} \prod_{h=0}^{t-1} S_{2 h}} \\
=\frac{1}{1+\frac{q_{2 t} N_{2} \prod_{h=0}^{t-1} S_{2 h}}{q_{1 t} N_{1} \prod_{h=0}^{t-1} S_{1 h}} .}
\end{gathered}
$$

If the ratio of catchabilities is constant over time, we can let $q=q_{2 t} N_{2} /\left(q_{1 t} N_{1}\right)$, thus obtaining

$$
\operatorname{Prop}(1, t)=\frac{1}{1+q \frac{\prod_{h=0}^{t-1} S_{2 h}}{\prod_{h=0}^{t-1} S_{1 h}} .}
$$

If the ratio $S_{2 h} / S_{1 h}$ is a constant, say $S$, over all time periods $h$, then equation (5) can be written as

$$
\operatorname{Prop}(1, t)=\frac{1}{1+q S^{t}}=\frac{1}{1+q e^{(\Delta Z) t}},
$$

where the parameter $S$ has been expressed as $S=$ $\exp (\Delta Z)$ to show the correspondence with a logistic model (see below). The parameter $\Delta Z$ represents the difference in the instantaneous rates of mortality for the two classes of animals (condition $1-$ condition 2). Here, $q$ subsumes the relative abundances of the two groups at the time of release and their relative catchabilities. Similarly, the proportion of animals recaptured at time $t$ from condition class 2 is

$$
\operatorname{Prop}(2, t)=1-\frac{1}{1+q e^{(\Delta Z) t}}=\frac{q e^{(\Delta Z) t}}{1+q e^{(\Delta Z) t}} .
$$

At this point, we can let $t$ be a continuous variable instead of an index of time intervals and treat equations (6) and (7) as the probabilities that a recaptured animal will be in a specified class given that a recapture has been made at time $t$. A formal derivation of these probabilities is given in Appendix 1.

The likelihood function can be constructed as the product of the probabilities for each recaptured animal. If there are $n_{i}$ recaptures of animals released in condition class $i(i=1,2)$ and the time until recapture of the $j$ th animal from condition class $i$ is denoted by $t_{i j}$, then, given the set of recapture times, the likelihood for all of the recaptures is given by 


$$
\Lambda=\prod_{j=1}^{n_{1}} \frac{1}{1+q e^{(\Delta Z) t_{1 j}}} \prod_{j=1}^{n_{2}} \frac{q e^{(\Delta Z) t_{2 j}}}{1+q e^{(\Delta Z) t_{2 j}}}
$$

(see Appendix 1 and Hoenig et al. 1990). The values of $q$ and $\Delta Z$ that maximize this function are the maximum likelihood estimates. The likelihood in equation (8) is the well-known logistic model.

The maximum likelihood estimate of the ratio $S$ can be obtained by back-transformation, that is, if $\Delta \hat{Z}$ is the maximum likelihood estimate of $\Delta Z$, then the estimate of $S$ is obtained from

$$
\hat{S}=\exp (\Delta \hat{Z})
$$

Note that valid estimates of $S$ or $\Delta Z$ can be obtained when the catchability of animals in condition 1 differs from that of animals in condition 2 provided the ratio of the catchabilities remains constant over time.

\section{A Model with Long- and Short-Term Effects}

Suppose now that equation (2) holds instead of equation (1). Then equation (4) becomes

$$
\operatorname{Prop}(1, t)=\frac{C_{1 t}}{C_{1 t}+\beta C_{2 t}},
$$

(where the expectations of catches are as defined in equation 3) and equation (8) becomes

$$
\Lambda=\prod_{j=1}^{n_{1}} \frac{1}{1+q^{*} e^{(\Delta Z) t_{1 j}-\delta}} \prod_{j=1}^{n_{2}} \frac{q^{*} e^{(\Delta Z) t_{2 j}-\delta}}{1+q^{*} e^{(\Delta Z) t_{2 j}-\delta}},
$$

where

$$
q^{*}=\frac{\beta q_{2} N_{2}}{q_{1} N_{1}} .
$$

The parameter $q^{*}$ is uninterpretable unless the ratio of catchabilities is known. Thus, for example, if the ratio of catchabilities is assumed to be 1.0, the maximum likelihood estimate of the relative survival over the initial period $\delta$ can be obtained as

$$
\hat{\beta}=\frac{\hat{q}^{*} N_{1}}{N_{2}},
$$

with $N_{2}$ and $N_{1}$ being the known number of releases in each condition class.

\section{A Model with Only Short-Term Effects}

Consider now the important case in which all released fish have the same survival rate after a recovery period of length $\delta$. In this case, the expected ratio of all recaptures obtained after time $\delta$ reflects the constant ratio of abundances of the two groups after the initial period of differential survival, provided the animals in the two condition classes have the same catchability after the recovery period $\delta$. The maximum likelihood estimates can then be found analytically as

$$
\hat{\beta}=\frac{C_{2} / N_{2}}{C_{1} / N_{1}}=\frac{R_{2}}{R_{1}},
$$

where $R_{1}$ is the ratio of the number of animals tagged in condition 2 to the number tagged in condition 1 and $R_{2}$ is the ratio of recaptures (condition 2 : condition 1 ) after time $\delta$. Equation (10) is the familiar relative risk used in the health sciences to compare health risks for two conditions or categories in terms of relative survival rates (see Rosner 1990).

A two-sided confidence interval can be obtained as

$$
\left(\hat{\beta} e^{-Z_{1-\alpha / 2} \sqrt{v}}, \hat{\beta} e^{Z_{1-\alpha / 2} \sqrt{v}}\right),
$$

where $Z_{1-\alpha / 2}$ is the $100(1-\alpha / 2)$ th percentage point of the standard normal distribution and

$$
v=\operatorname{var}\left(\log _{e} \hat{\beta}\right)=\frac{N_{1}-C_{1}}{N_{1} C_{1}}+\frac{N_{2}-C_{2}}{N_{2} C_{2}}
$$

(SAS Institute 1989). The computations are easily accomplished using the SAS statistical package (Appendix 2).

\section{An Example of Applying the Short-Term-Effects Model: Sharks Released from Gill Nets}

To illustrate the efficacy of the method, we now apply the short-term-effects model to the case of coastal sharks caught in gill nets. In the latter half of the 20th century until 1994, commercial gill-net fisheries targeting mullet Mugil spp., Florida pompano Trachinotus carolinus, and other teleost species operated along the Gulf of Mexico coast of Florida. To quantify the amount and fates of shark bycatch in this gear type, Mote Marine Laboratory's Center for Shark Research (CSR) began fisheries-independent surveys in Florida Gulf coastal waters in 1992 by deploying gill nets, quantifying the catch and its condition in the nets, and tagging and releasing the surviving sharks. Although Florida banned the use of gill nets by fishermen in 1994, the CSR's scientific use of gill nets continued through 2004 as part of a comprehensive research program on shark relative abundance, nursery areas, and migratory behavior (Hueter and Tyminski, in press).

In most of these scientific collections, monofilament gill nets with a stretch mesh of $11.4 \mathrm{~cm}$, a height of 3 $\mathrm{m}$, and a length of approximately $366 \mathrm{~m}$ were deployed. This gear type has relatively high selectivity for small sharks and relatively low bycatch of other species in Florida coastal waters. The nets had a bottom lead line and top float line and typically were anchored at both ends. In most deployments, the net was allowed to soak for approximately $1 \mathrm{~h}$ (from first mesh in to last mesh out) prior to retrieval, but other set times sometimes were used in exploratory surveys. All catch was documented and sharks were identified, sexed, 
TABLE 1.-Description of condition categories for released sharks.

\begin{tabular}{|c|c|}
\hline Condition & Criteria \\
\hline 1 (Good) & $\begin{array}{l}\text { No revival time required when shark is returned to the water; } \\
\text { rapid swimming away on release, usually with a vigorous splash. }\end{array}$ \\
\hline 2 (Fair) & No revival time required; slow but strong swimming away on release. \\
\hline 3 (Poor) & $\begin{array}{l}\text { Short revival time (up to } 30 \mathrm{~s} \text { ) required; once revived, slow } \\
\text { but sometimes atypical swimming away on release. }\end{array}$ \\
\hline 4 (Very poor) & $\begin{array}{l}\text { Long revival time (more than } 30 \mathrm{~s} \text { ); once revived, limited } \\
\text { or no swimming observed on release but respiration functional. }\end{array}$ \\
\hline 5 (Dead) & $\begin{array}{l}\text { Dead on removal from gear or moribund and unable to revive } \\
\text { even after a long resuscitation time. }\end{array}$ \\
\hline
\end{tabular}

measured in length to the nearest $\mathrm{cm}$, weighed to the nearest $0.1 \mathrm{~kg}$, and either tagged and released or retained for study. Live sharks were tagged with a nylon-head, plastic barb tag (Hallprint Pty. Ltd., Victor Harbor, Australia) inserted just below the first dorsal fin across the body midline, such that the tag head was firmly anchored in the cartilage and connective tissue below the fin.

When a tagged shark was released, the general condition of the fish was assessed according to the criteria described in Table 1. Four classes of release condition ranging from the best (condition 1) to the worst (condition 4) were used; sharks that were dead at the boat were classified as being in condition 5 . The judgment criteria are relatively objective and emerged from years of observing sharks caught in a variety of gear types. More physiologically based criteria can be used (see Manire et al. 2001), but this behavioral scoring is simple and rapid, which is beneficial in tagging and bycatch survival research, where the goal is to release the most fish in the best possible condition.

To test the efficacy of our method, we focus here on the results for the two species with the greatest number of tag returns, the blacktip shark Carcharhinus limbatus and the bonnethead Sphyrna tiburo, which had tag return rates of $125 / 2,898=4.3 \%$ and $155 / 4,352$ $=3.6 \%$, respectively (Table 2 ). The number of tag returns was insufficient to fit the two-parameter logistic model (equation 9). Consequently, we estimated the ratio of survival rates over the recovery period immediately after release of the tagged sharks (equation 10).

In doing so, we made three assumptions. First, there is no long-term differential in the mortality of sharks that survive the capture-tag-release event. That is, regardless of release condition, those sharks that recovered from the event went on to experience comparable rates of mortality. Second, the subsequent catchability of the surviving sharks - as well as the probability that a recaptured, tagged shark will be reported-is equal regardless of release condition. Third, any artifacts of tagging (tag shedding, taginduced mortality, etc.) are equal across release conditions. Based on laboratory studies by the CSR, it is reasonable to assume that in the short term of several days after tagging, properly applied tags of the type used in our example case were not shed and did not cause fatal wounds. In the long term, our method remains valid as long as tag artifacts are the same across all release conditions. To date, recaptures of sharks tagged by the CSR with this type of tag have been recorded up to 8.7 years after release (Hueter and Tyminski, unpublished data).

The shortest time at liberty between tagging and

TABLE 2.- Stretch total lengths (STLs) of tagged blacktip sharks and bonnetheads by condition class on release.

\begin{tabular}{ccccc}
\hline & \multirow{2}{*}{$\begin{array}{c}\text { Number } \\
\text { of sharks }\end{array}$} & Minimum & Maximum & Mean \\
\cline { 3 - 5 } Condition & & Blacktip sharks & \\
& 928 & 52 & 144 & 66.6 \\
1 & 939 & 49 & 130 & 66.5 \\
2 & 666 & 53 & 154 & 68.6 \\
3 & 365 & 53 & 114 & 89.3 \\
4 & 2,898 & & & 81.1 \\
Total & 723 & 42 & 116 & 80.2 \\
& 1,355 & 42 & 113 & 79.9 \\
2 & 1,383 & 41 & 112 & \\
3 & 891 & & & \\
4 & 4,352 & & & \\
Total & & & & \\
\end{tabular}


TABLE 3.- Release and recapture data for blacktip sharks and bonnetheads, by condition.

\begin{tabular}{crrrrr}
\hline & \multicolumn{2}{c}{ Blacktip sharks } & & \multicolumn{2}{c}{ Bonnetheads } \\
\cline { 2 - 3 } \cline { 5 - 6 } Condition & $\begin{array}{c}\text { Number } \\
\text { tagged }^{\mathrm{a}}\end{array}$ & $\begin{array}{c}\text { Number } \\
\text { recaptured (\%) }\end{array}$ & & $\begin{array}{c}\text { Number } \\
\text { tagged }^{\mathrm{a}}\end{array}$ & $\begin{array}{c}\text { Number } \\
\text { recaptured (\%) }\end{array}$ \\
\hline 1 & 928 & $58(6.3)$ & 723 & $43(6.0)$ \\
2 & 939 & $39(4.2)$ & 1,355 & $65(4.8)$ \\
3 & 666 & $24(3.6)$ & 1,383 & $36(2.6)$ \\
4 & 365 & $4(1.1)$ & 891 & $11(1.2)$ \\
5 & {$[1,898]$} & & {$[2,558]$} & \\
\hline
\end{tabular}

${ }^{\mathrm{a}}$ Animals in condition 5 were not tagged; the number identified is shown in brackets.

recapture was $4 \mathrm{~d}$ for blacktip sharks and $2 \mathrm{~d}$ for bonnetheads. Consequently, by using all of the recapture data we are implicitly assuming that the period of differential mortality (i.e., the recovery period) ends by 4 and $2 \mathrm{~d}$ after the catch-tag-release event for the two species, respectively. This assumption was checked by progressively eliminating recaptures with short times at liberty and noting the degree to which the estimates changed.

To check on the possibility that condition class was confounded with the size of the animals, we computed the mean size of the animals tagged and released in each condition class.

\section{Results}

The sizes of the released animals varied little among condition classes (Table 2). Therefore, size was not confounded with condition class in the study.

Inspection of the data in Table 3 reveals that the percentage of recaptured sharks declined with worsening condition category for both species, suggesting that survival rate immediately following the catch-tag-release event did depend on the condition at release. Equations (10) and (11) were applied successively to the data in Table 3 for conditions 2 , 3 , and 4 to estimate the survival rates relative to that for condition 1 (Table 4). In the case of blacktip sharks, the survival of condition- 2 animals was about two-thirds that of condition-1 animals, while for bonnetheads the ratio was 0.81 . The survival of condition-3 animals was lower than that of condition-
2 animals for both species, survival relative to that of condition- 1 animals being 0.58 and 0.44 for blacktip sharks and bonnetheads, respectively. The estimated survival of condition-4 animals was low (18-21\%) for both species.

Estimates of relative survival were computed after removing all recaptures within $k$ days of tagging, for $k$ $=2,3, \ldots, 6$. None of the estimates changed appreciably, the maximum change being less than $11 \%$. Increasing the value of $k$ tends to reduce the number of recaptured fish used. Consequently, we take as our estimates those based on all of the data.

To determine the absolute postrelease (cryptic) mortality rates of sharks as a result of the catch-tagrelease event, we made the assumption that all of the animals released in the very best condition (condition 1) recovered from the event and subsequently had the normal survival rate for wild sharks. In this case, the relative survival rates become absolute rates. In our example, it is estimated that the survival rate of blacktip sharks in condition 2 (relative to that of sharks in condition 1) was 0.66 (Table 4 ). If all condition-1 sharks survived the event and went on to experience the normal survival rate during the period of length $\delta$ after release (presumably close to $100 \%$ because $\delta$ is a short period of time), then the percentage of condition-2 blacktip sharks that survived the catchtag-release event was $66 \%$.

This allows us to estimate the fraction of the releases that did not survive the tagging event by simply multiplying the number released in each condition class

TABLE 4.-Estimates of the survival of blacktip sharks and bonnetheads in conditions 2-4 relative to that of the same species in condition $1 ; \hat{\beta}$ is the estimated ratio of survival rates. The estimates are based on equations (10) and (11) in the text; $\mathrm{CI}=$ the $95 \%$ confidence interval.

\begin{tabular}{ccccc}
\hline & \multicolumn{2}{c}{ Blacktip sharks } & & \multicolumn{2}{c}{ Bonnetheads } \\
\cline { 2 - 3 } \cline { 5 - 5 } Condition & $\hat{\beta}$ & CI & $\hat{\beta}$ & CI \\
\hline 2 & 0.66 & $0.45-0.99$ & 0.81 & $0.55-1.17$ \\
3 & 0.58 & $0.36-0.92$ & 0.44 & $0.28-0.68$ \\
4 & 0.18 & $0.06-0.48$ & 0.21 & $0.11-0.40$ \\
\hline
\end{tabular}


TABLE 5.-Cryptic postrelease mortality of sharks released from gill nets. All sharks in condition 1 were assumed to have survived the catch-tag-release event. For blacktip sharks, 31\% (898 of 2,898) of released sharks are estimated to have died from the event; for bonnetheads, the number is $40 \%(1,735$ of 4,352$)$.

\begin{tabular}{|c|c|c|c|}
\hline Condition & Number tagged (\%) & Survival & Number dying (\%) \\
\hline \multicolumn{4}{|c|}{ Blacktip sharks } \\
\hline 1 & $928(32)$ & 1.00 & $0(0)$ \\
\hline 2 & $939(32)$ & 0.66 & $319(34)$ \\
\hline 3 & $666(23)$ & 0.58 & $280(42)$ \\
\hline 4 & $365(13)$ & 0.18 & $299(82)$ \\
\hline Total & 2,898 & & 898 (31) \\
\hline \multicolumn{4}{|c|}{ Bonnetheads } \\
\hline 1 & $723(17)$ & 1.00 & $0(0)$ \\
\hline 2 & $1,355(31)$ & 0.81 & 257 (19) \\
\hline 3 & $1,383(32)$ & 0.44 & $774(56)$ \\
\hline 4 & $891(20)$ & 0.21 & $704(79)$ \\
\hline Total & 4,352 & & $1,735(40)$ \\
\hline
\end{tabular}

by the corresponding mortality rate $(1-$ the survival rate) and summing over all condition classes (Table 5). Applying this to the 1992-2004 gill-net data, we estimate that $31 \%$ of the released blacktip sharks and $40 \%$ of the bonnetheads died as a direct result of the catch-tag-release event. In other words, $69 \%$ of the tagged blacktip sharks and $60 \%$ of the tagged bonnetheads survived the initial stress of capture and release and went on to experience the normal survival rate in the wild. Adding the immediate, at-the-boat mortality (condition-5 animals) of $40 \%$ of the catch for blacktip sharks and $37 \%$ for bonnetheads results in a total episodic mortality of $58 \%$ and $62 \%$ for the two species, respectively, as a consequence of being caught in the gill net and, if alive, tagged and released.

\section{Discussion}

The estimates of high postrelease cryptic mortality in our example illustrate the need for methods to quantify this source of mortality in fisheries and fisheries research. Our approach to quantifying release or discard mortality is general in nature and based on the established statistical theory of the logistic model. Thus, when there are ample data, it is possible to generalize the model, such as through the introduction of covariates. It is also possible to linearize the model and determine goodness of fit by examining residuals. If the ratio of recaptures, $R_{T}$, is computed over a series of discrete time intervals, then from equation (2) a plot of $\log \left(R_{T}\right)$ versus time interval, $T$, will produce a linear relationship with a slope estimating $\log \left(S_{2} / S_{1}\right)$. The intercept estimates the log of the product of $\beta, R$, and the ratio of catchabilities, which is useful if one can assume a value for the ratio of catchabilities.

The modeling of catchabilities is a bit complicated because there are several cases to consider. If there is only a long-term (chronic) effect, we do not need the catchabilities of the two classes to be equal; we only need their ratio to remain constant over time. This is because, in a plot of the log of the ratio of catches versus time, the intercept measures the catchabilities and the slope measures the difference in mortality rates independently of the catchabilities.

If there is only an initial effect, then we require the ratio of catchabilities to be 1.0 , but only after some point in time $\delta$. This is because we are comparing the known ratio of the abundances of the animals that were tagged with the ratio of the survivors as seen in the tag returns. The catchabilities of the two groups affect the ratio of recaptures. For example, consider a case in which near-moribund fish are less likely to swim into a gill net. The model with only a long-term effect is appropriate if the near-moribund fish always have the same catchability regardless of elapsed time. Similarly, if they have a low catchability during time period $\delta$ and after that are either dead or completely recovered (so that their catchability becomes the same as for condition-1 fish), then we would still be able to use the initial-effect-only model.

The situation becomes more complicated when the ratio of catchabilities changes over time. Such situations can be handled in models of multiple releases of tagged fish at different times (see Burnham et al. 1987) but are intractable when a single release is made.

As a practical matter, to get a good estimate of the intercept it is necessary to obtain some tag returns shortly after the tagging episode. To get a good estimate of the relative mortality rate (slope), it is necessary to obtain recaptures at points well separated in time (so that the effects of the differential mortality will be expressed). This suggests a study design in which concerted efforts are made to obtain tag returns shortly after tagging and again a year later (such that the differential mortality operates over the entire annual cycle). Additional sampling events would be helpful for testing assumptions, such as whether the differential mortality is constant.

If the animals in condition 1 experience some 
mortality from being captured, tagged, and released, the procedure for estimating absolute survival rates will be biased upward for all condition classes. For example, if there is tagging-induced mortality in a study of bycatch survivorship, the amount of discard mortality will be underestimated. In cases such as this, managers using these estimates need to understand that there is potential for underestimating discard mortality with this technique. To minimize bias, a number of steps can be taken. First, the condition-1 category should be limited strictly to fish showing negligible deleterious effects of the capture event upon release. Second, assignment to the other condition categories should be based on observable, repeatable, and consistent criteria, as in Table 1. Third, the tags that are used for these studies should have minimal effects on the health, behavior, and survivorship of the tagged fish.

In some cases, there may not be a condition class 1 for which survival can reasonably be assumed to be close to that of normal fish that have not been captured. An example is a species with a physoclistous swim bladder that resides at great depth. Our method is not suitable for estimating absolute survival in such cases. Other methods, such as acoustic and pop-up satellite tags, may be more useful for studying the survival of those fish.

Our approach to estimating postrelease survival is adaptable to any fishery or research project for which a tag-recapture program is in place and animal condition at release can be categorized. Any number of release condition categories can be used, as long as there are sufficient sample sizes in each category. The models can be generalized to include multiple (e.g., annual) releases of tagged fish. A model with explicit year effects can be used to test more assumptions and to estimate year effects and the effects of factors such as water temperature. The reader is referred to Burnham et al. (1987) for model development.

This method does not replace other approaches to estimating postrelease mortality that use telemetry, cage studies, and the like. Instead, our approach adds to the toolbox of available research techniques with which to determine survivorship in tagging programs and the impact of release or discard mortality on fish populations. Although we used a shark example here, our method is applicable to most fish that can be classified into condition categories and released as part of a tag-recapture program.

\section{Acknowledgments}

We thank the staff members and volunteer student interns of Mote Marine Laboratory's Center for Shark
Research who conducted the field research that contributed to this work. Special gratitude is extended to the cooperating recreational and commercial fishermen who reported shark tag recaptures. We thank Dennis Heisey of the U.S. Geological Survey, National Wildlife Health Center, in Madison, Wisconsin, and Kenneth Pollock of North Carolina State University for helpful discussions. This work was supported by the Florida Department of Natural Resources (now the Florida Fish and Wildlife Conservation Commission), the NOAA/NMFS MARFIN Program, the NOAA/ NMFS Highly Migratory Species Division, and NOAA/NMFS through the National Shark Research Consortium. This is VIMS contribution 2723.

\section{References}

Alverson, D. L., M. H. Freeberg, S. A. Murawski, and J. G. Pope. 1994. A global assessment of fisheries bycatch and discards. Food and Agriculture Organization of the United Nations, Fisheries Technical Paper 339, Rome.

Burnham, K. P., D. R. Anderson, G. C. White, C. Brownie, and K. H. Pollock. 1987. Design and analysis methods for fish survival experiments based on release-recapture. American Fisheries Society, Monograph 5, Bethesda, Maryland.

Heupel, M. R., and C. A. Simpfendorfer. 2002. Estimation of survival and mortality of juvenile blacktip sharks, Carcharhinus limbatus, within a nursery area based on telemetry data. Canadian Journal of Fisheries and Aquatic Sciences 59:624-632.

Hoenig, J. M., P. Pepin, and W. D. Lawing. 1990. Estimating relative survival rate for two groups of larval fishes from field data: do older larvae survive better than young? Fishery Bulletin 88:485-491.

Hueter, R. E., and J. P. Tyminski. In press. Species-specific distribution and habitat characteristics of shark nurseries in Gulf of Mexico waters off peninsular Florida and Texas. In C. McCandless, N. Kohler, and H. L. Pratt, editors. Shark nursery grounds of the Gulf of Mexico and the east coast of the United States. American Fisheries Society, Special Publication, Bethesda, Maryland.

Keniry, M. J., W. Brofka, W. H. Horns, and J. E. Marsden. 1996. Effects of decompression and puncturing the gas bladder on survival of tagged yellow perch. North American Journal of Fisheries Management 16:201-206.

Manire, C., R. Hueter, E. Hull, and R. Spieler. 2001. Serological changes associated with gill-net capture and restraint in three species of sharks. Transactions of the American Fisheries Society 130:1038-1048.

Ricker, W. E. 1975. Computation and interpretation of biological statistics of fish populations. Fisheries Research Board of Canada Bulletin 191.

Rosner, B. 1990. Fundamentals of biostatistics, 3rd edition. P.W.S.-Kent Publishing Company, Boston.

SAS Institute. 1989. SAS/STAT user's guide, version 6, 4th edition, volume 1. SAS Institute, Inc., Cary, North Carolina. 


\section{Appendix 1: Derivation of the Likelihood in Equation (8)}

We begin by considering a discrete-time tag-return model as described, for example, by Brownie et al. (1985). Recaptures over time from each condition class $i$ are assumed to be samples from independent multinomial distributions with parameters $\vec{\pi}_{i}=\left[\pi_{i 1}\right.$, $\left.\pi_{i 2}, \ldots, \pi_{i p}, \pi_{i, p+1}\right]^{T}$, where $\pi_{i j}$ is the probability that an animal tagged in condition $i$ will be recaptured in time period $j(j=1,2 \ldots, p)$ and $\pi_{i, p+1}$ is the probability that an animal tagged in condition $i$ will not be seen again during the course of the study. Clearly, since the probabilities must sum to 1.0 ,

$$
\pi_{i, p+1}=1-\sum_{j=1}^{p} \pi_{i j} .
$$

When $\pi_{i, p+1}$ approaches 1.0 for both condition classes (i.e., most tags are never recovered), the distributions of the catches in the $p$ time periods can be approximated by independent Poisson random variables with parameters $\pi_{i j}$. The Poisson parameters for condition 1 are as follows:

$$
\begin{array}{ll}
\text { Time period 1 } & \theta_{1} \\
\text { Time period 2 } & \theta_{2} \cdot \exp \left(-Z_{1}\right) \\
\text { Time period } 3 & \theta_{3} \cdot \exp \left(-Z_{1}-Z_{2}\right), \cdots,
\end{array}
$$

where $\theta_{j}=q_{j} N_{1}$ is the tag recovery rate with $q_{j}$ being the period-specific catchability for condition 1 and $N_{1}$ being the number tagged in condition 1 ; the $\mathrm{Zs}$ are periodspecific total instantaneous mortality rates for condition 1 .

For animals in condition 2, the parameters have the same structure as for condition 1 but the $\theta$ s are assumed to be a constant multiple of the $\theta$ s for condition 1 and the total mortalities are assumed to differ from those of condition 1 by a constant $\Delta Z$. Thus, the parameters for condition 2 are

$$
\begin{array}{ll}
\text { Time period 1 } & \gamma \theta_{1} \\
\text { Time period 2 } & \gamma \theta_{2} \cdot \exp \left(-Z_{1}-\Delta Z\right) \\
\text { Time period 3 } & \gamma \theta_{3} \cdot \exp \left(-Z_{1}-Z_{2}-2 \Delta Z\right), \cdots .
\end{array}
$$

For two independent Poisson random variables, $X$ and $Y$, with parameters $\lambda_{x}$ and $\lambda_{y}$, respectively, the distribution of $X$ given the sum of $X$ and $Y$ is binomial with parameter $\lambda_{x} /\left(\lambda_{x}+\lambda_{y}\right)$ (Johnson and Kotz 1969). Thus, the catch of condition- 1 animals in a time period $j$ given the total catch in period $j$ (condition 1 and condition 2 combined) is binomial with parameter $\pi_{1 j} /\left(\pi_{1 j}+\pi_{2 j}\right)$. Note that the $\theta$ s and Zs cancel out of the binomial parameters.

Finally, shrinking the durations of the time periods to 0 gives the product Bernoulli likelihood in equation (8).

Burnham et al. (1987; section on first capture histories models) develop estimators of the relative survival rate directly from multinomial distributions for each of the condition classes and thus avoid the Poisson approximation. Their important work should be consulted for additional models and hypothesis tests.

\section{References}

Brownie, C., D. R. Anderson, K. P. Burnham, and D. S. Robson. 1985. Statistical inference from band recovery data: a handbook. U.S. Fish and Wildlife Service Resource Publication 156.

Burnham, K. P., D. R. Anderson, G. C. White, C. Brownie, and K. H. Pollock. 1987. Design and analysis methods for fish survival experiments based on release-recapture. American Fisheries Society, Monograph 5, Bethesda, Maryland.

Johnson, N. L., and S. Kotz. 1969. Discrete distributions. Houghton Mifflin, Boston.

\section{Appendix 2: Computing Estimates of Relative Risk and Confidence Intervals Using SAS}

Estimates of relative risk and the associated confidence limits can be obtained from tagging data by using the freq procedure in SAS (SAS Institute 1989). The data are arranged in a $2 \times 2$ table as in

Table A.2.1. The following instructions are used:

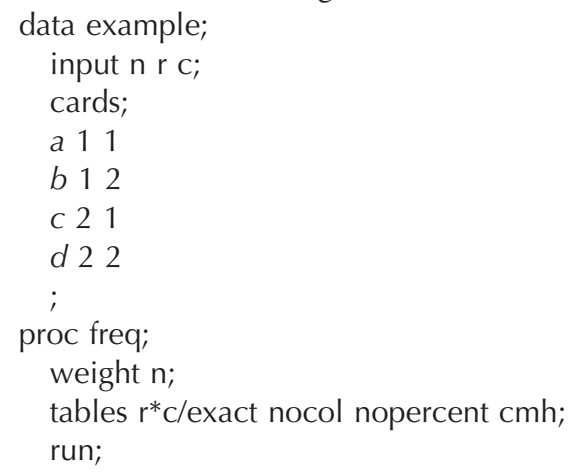

In the above, $a, b, c$, and $d$ are replaced by the actual values (data).
The appropriate part of the output is labeled cohort (col 1 risk). Two types of confidence interval are given: Mantel-Haenszel and logit. The latter tend to be slightly wider.

TABLE A.2.1.-Data specification for estimating relative risk. Variables are defined as follows: $N_{1}, N_{2}=$ the number of animals tagged and released in condition 1 and condition 2 , respectively; $C_{1}, C_{2}=$ the number of those animals subsequently recaptured.

\begin{tabular}{lcc}
\hline & \multicolumn{2}{c}{ Number of animals } \\
\cline { 2 - 3 } Condition & Recaptured & Not recaptured \\
\hline 2 & $a=C_{2}$ & $b=N_{2}-C_{2}$ \\
1 & $c=C_{1}$ & $d=N_{1}-C_{1}$ \\
\hline
\end{tabular}

\section{Reference}

SAS Institute. 1989. SAS/STAT user's guide, version 6, 4th edition, volume 1. SAS Institute, Inc., Cary, North Carolina. 